\title{
Buffering and Trapping Ultrashort Optical Pulses in Concatenated Bragg Gratings
}

\author{
Shenhe $\mathrm{Fu}^{1}$, Yikun Liu ${ }^{\dagger, 1}$ Yongyao $\mathrm{Li}^{2,3}$, Liyan Song, ${ }^{1}$ Juntao Li, ${ }^{1}$ Boris A. Malomed, ${ }^{3}$ and Jianying Zhou ${ }^{1}$ \\ ${ }^{1}$ State Key Laboratory of Optoelectronic Materials and Technologies, \\ Sun Yat-sen University, Guangzhou 510275, China \\ ${ }^{2}$ Department of Applied Physics, South China Agriculture University, Guangzhou 510642, China \\ ${ }^{3}$ Department of Physical Electronics, School of Electrical Engineering, \\ Faculty of Engineering, Tel Aviv University, Tel Aviv 69978, Israel \\ †Corresponding author: ykliu714@gmail.com
}

\begin{abstract}
Strong retardation of ultrashort optical pulses, including their deceleration and stoppage in the form of Bragg solitons in a cascaded Bragg-grating (BG) structure, is proposed. The manipulations of the pulses are carried out, using nonlinear effects, in a chirped BG segment which is linked, via a defect, to a uniform grating. The storage of the ultrashort pulses is shown to be very robust with respect to variations of the input field intensity, suggesting the feasibility of storing ultrafast optical pulses in such a structure. Physical estimates are produced for the BGs written in silicon.

OCIS codes: $060.3735,060.1810,190.5530$
\end{abstract}

Using the slow or stopped light to buffer optical signals has drawn a great deal of attention [1 3$]$ due to potential applications to optical telecommunication and signal processing, as well as implications for studies of fundamental properties of optical pulses propagating in complex media. Various schemes have been proposed for making slow light, including electromagnetically induced transparency [4 [6], photonic-crystal waveguides [7-10], resonantly-absorbing gratings [1, 11-13] and resonant fiber loops [3], among others [14, 15]. However, for high-speed signal processing and communications operating with short input pulses at high repetition rates [16, 17], the design of optical-signal buffering is still a challenging problem.

In this context, designing devices for the generation of slow light on the basis of Bragg-grating (BG) structures [2, 18 22] is of great interest due to their tunability and cascadability. In such periodic structures, strong dispersion of light is induced in the vicinity of the photonic bandgap [2]. Mobile and immobile BG solitons were predicted theoretically long ago [20, 23]. A possibility of their retardation using apodized gratings 24], as well as producing standing soliton-like pulses as a result of the collisions of counterpropagating moving ones [25], were analyzed in detail too. Experimentally, BG solitons were first created with the velocity at $75 \%$ of the speed of light in the uniform optical fiber in the cladding of which the BG was written 21]. Later, slow robust light pulses traveling at $16 \%$ [2] and 19\% 26] of this speed were reported. Theoretical and experimental studies of slow light in chirped BGs 27] and superstructures [28] have also been performed. Nevertheless, standing or very slow light in BGs has not been reported yet, which remains a fundamental issue.

Generally, two conditions should be met for the creation of very slow BG solitons. Firstly, the gratinginduced dispersion must be balanced by the Kerr nonlinearity [29]. Secondly, since the optical field is represented by forward- and backward-traveling waves in the BG, an initial configuration should be built with the nearly equal powers of the two waves [30]. The former condition can be achieved using a high-power input, whose nonlinearity is strong enough [21]. The latter condition is much harder to meet with the single incident pulse [31], as it does not initially contain any backward component.

The objective of this work is to explore possibilities for the generation of slow ultrashort pulses, or even standing ones, in concatenated BGs, built as a linearly chirped grating which is linked, through a defect, to a uniform one. Essential advantages of this setting are demonstrated below. Firstly, initial conditions for pulses at the input edge of the uniform-BG segment may be manipulated by means of the preceding chirped BG, which provides a possibility of preparing the right mix of the forward and backward fields. Secondly, stable standing light pulses trapped by the defect at the junction between the chirped and uniform segments are found in a broad interval of values of the light intensities. Without the defect, the stopped pulses turn out to be unstable.

We assume that the period of the chirped BG decreases continuously along the propagation distance, while the period of the uniform BG is fixed to be equal to its value at the output edge of the chirped BG. Accordingly, the nominal Bragg wavelength is gradually shifted to smaller values for the pulse propagating along the chirped BG, hence the wavelength of the input pulse, originally taken near the blue edge of the bandgap, will be slightly shifted into the depth of the bandgap. As a result, a fraction of the power of the forward field component is converted into the backward field, leading to a decrease of the pulse's velocity. Selecting an appropriate chirp, and making use of the Kerr nonlinearity, it is possible to create conditions for the generation of very slow or even immobile pulses.

First, we consider the setting without the grating defect, hence the linear refractive index of such a tailored BG takes the form of [32] $n(z)=$ $n_{0}\left[1+2 \Delta n \cos \left(2 \pi z(1+C z) / \Lambda_{0}\right)\right]$ for $0 \leq z<L / 8$, and $n(z)=n_{0}\left[1+2 \Delta n \cos \left(2 \pi z(1+(1 / 8) C L) / \Lambda_{0}\right)\right]$ for $L / 8 \leq z \leq L$, where $z$ is the propagation distance, $L$ the total length of the grating, $n_{0}$ the average refractive index, $\Delta n$ its modulation depth, $\Lambda_{0}$ the BG period at the 
input edge, and $C$ is the chirp. The implication of this setting is that the length of the chirped segment is $1 / 8$ of the total length. As said above, the chirped segment is introduced for manipulating the form of the pulse arriving at the input edge of the uniform grating.

The standard coupled-mode theory [29, 32] for the light propagation in uniform BGs can be applied as well to chirped gratings 27] and Bragg superstructures [28]. For the slowly varying envelopes of forward and backward waves, $E_{f}$ and $E_{b}$, the coupled-mode equations are written as 32 ]

$$
\begin{gathered}
\pm i \frac{\partial E_{f, b}}{\partial z}+\frac{i}{v_{g}} \frac{\partial E_{f, b}}{\partial t}+\delta(z) E_{f, b}+\kappa(z) E_{b, f} \\
+\gamma\left(\left|E_{f, b}\right|^{2}+2\left|E_{b, f}\right|^{2}\right) E_{f, b}=0
\end{gathered}
$$

where $t$ is time, $v_{g}=c / n_{0}$ is the group velocity in the material of which the BG is fabricated, $c$ is the speed of light in vacuum, $\gamma=n_{2} \omega / c$ is the nonlinearity strength, with $\omega$ being the frequency of the carrier wave and $n_{2}$ the Kerr coefficient. The wavenumber detuning parameter in Eqs. (1), corresponding to the adopted BG profile, is $\delta(z)=\Delta-2 \pi C z / \Lambda_{0}$ for $0 \leq z<L / 8$, and $\delta(z)=\Delta-2 \pi C L /\left(8 \Lambda_{0}\right)$ for $L / 8 \leq z \leq L$, where $\Delta \equiv 2 \pi n_{0} / \lambda-\pi / \Lambda_{0}$, while $\kappa=\pi \Delta n / \Lambda_{0}$ represents the coupling between the forward and backward fields.

The variation of the wavenumber-detuning $\delta(z)$, as a function of the propagation distance, $z$, is shown in Fig. 1(a). If Eqs. (1) are considered as coupled Schrödinger equations, the corresponding effective potential energy $V(z)$ is proportional to $-\delta(z)$ [as shown in Fig. 1(b)], which may be used for the qualitative analysis of the motion of the pulse treated as a quasiparticle [33]. Accordingly, if the initial kinetic energy of the quasiparticle, $V$, is smaller than the asymptotic level $V_{0}$, the moving pulse bounces back, as illustrated in the top plot of Fig. 11(c). With the initial energy $V=V_{0}$ [the middle plot in Fig. 11(c], the pulse nearly comes to a halt, which is explained by the character of the coupling between the forward and backward fields in the chirped BG, and its interplay with the Kerr nonlinearity. Finally, a pulse slowly advancing into the uniform BG can be obtained for the initial energy slightly exceeding $V_{0}$ [the bottom plot in Fig. 1(c)]. Below, these three options are confirmed by systematic simulation of Eqs. (11).

The simulations were performed with physical parameters of silicon, whose self-focusing-Kerr coefficient is $\left.4.5 \times 10^{-14} \mathrm{~cm}^{2} / \mathrm{W} 34\right]$ [here, we neglect the two-photon absorption (TPA) effect, which is essential in silicon [35], and is included below], and the average refractive index is $n_{0}=3.42$. The refractive-index modulation depth and chirp are taken as $\Delta n=0.006$ and $C=2.5064 \times 10^{-4}$ $\mathrm{cm}^{-1}$, respectively. The sufficient total length $L$ is 1.5 $\mathrm{cm}$. At the input edge of the sample, the BG period is fixed to be $\Lambda_{0}=154.1 \mathrm{~nm}$, and the Gaussian pulse, of temporal width $16 \mathrm{ps}$, is coupled into the system at carrier wavelength $1053 \mathrm{~nm}$.

With these parameters, simulations of Eq. (1) produce the results displayed in Fig. 1(d). From these re-

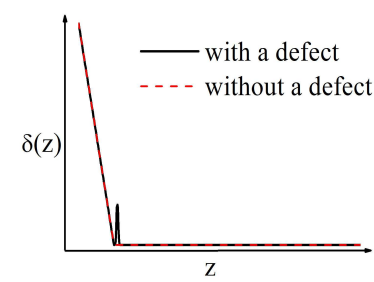

(a)

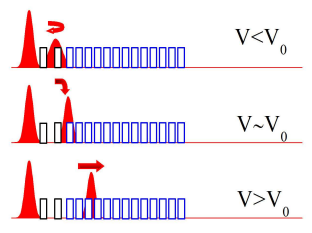

(c)

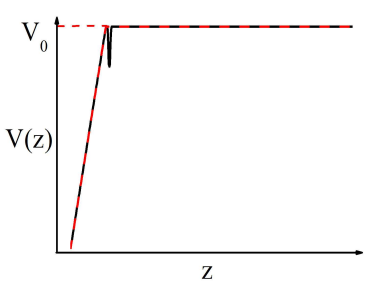

(b)

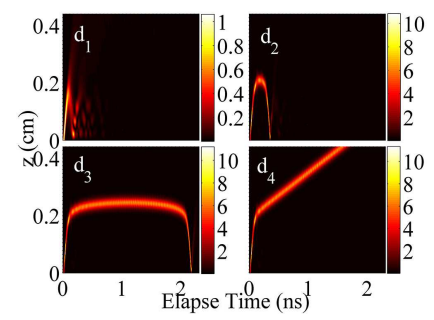

(d)
FIG. 1. (Color online) (a) The wavenumber detuning as a function of the propagation distance. (b) The dependence of the effective potential energy on the propagation distance, which is reverse to that of the detuning. (c) A sketch of the pulse propagation in the gratings without the defect, in three different cases. (d) Simulations of the pulse propagation for different peak intensities, injected into the grating without the defect: $\left(d_{1}\right) 0.65 \mathrm{GW} / \mathrm{cm}^{2},\left(d_{2}\right) 2.26 \mathrm{GW} / \mathrm{cm}^{2},\left(d_{3}\right) 2.29$ $\mathrm{GW} / \mathrm{cm}^{2},\left(d_{4}\right) 2.30 \mathrm{GW} / \mathrm{cm}^{2}$.

sults, three conclusions can be made, varying the tunable input-pulse peak intensity, $I_{P}$ - first, in the absence of the inserted defect.

(i) At low intensities, such as $I_{P}=0.65 \mathrm{GW} / \mathrm{cm}^{2}$ [panel $\left(d_{1}\right)$ in Fig. 1(d)], the pulse is almost totally reflected by the photonic bandgap, conspicuously broadening in the course of the propagation through the chirped BG, i.e., the pulse's dispersion is not compensated by the weak nonlinearity.

(ii) An unstable standing light pulse trapped at the interface is generated at higher intensities. In particular, at $I_{P}=2.26 \mathrm{GW} / \mathrm{cm}^{2}$, the pulse stops for a while and is then reflected, as seen in panel $\mathrm{d}_{2}$ of Fig. 1, Slightly increasing $I_{P}$ to $2.29 \mathrm{GW} / \mathrm{cm}^{2}$ in panel $\mathrm{d}_{3}$, it is observed that the pulse halts at the interface for almost $1.3 \mathrm{~ns}$, but eventually shows its intention to start the reverse motion. The pulse's peak intensities in this case, ranging from $2.26 \mathrm{GW} / \mathrm{cm}^{2}$ to $2.29 \mathrm{GW} / \mathrm{cm}^{2}$, slightly change in the course of the evolution.

(iii) A very slow pulse moving into the uniform BG, which may be identified as a BG soliton, can be created, as shown in panel $\mathrm{d}_{4}$, for $I_{P}=2.30 \mathrm{GW} / \mathrm{cm}^{2}$. In this case, the pulse is propagating in the uniform BG with a very small velocity, $0.005 c$ (which is much smaller than the results obtained in Refs. [2, 21, 26]). Naturally, the simulations demonstrate that the velocity of the moving soliton increases with the further increase of $I_{P}$.

Since defects may help in trapping light in the Bragg 


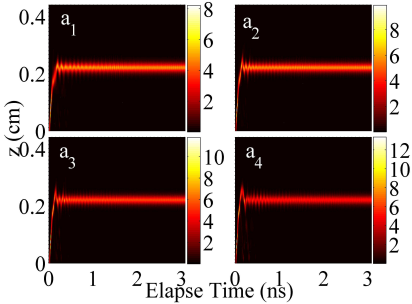

(a)

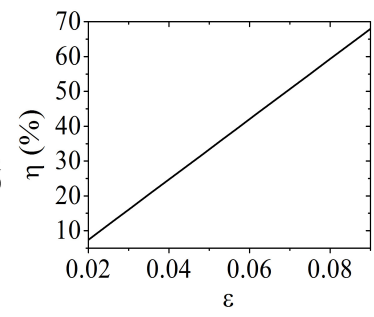

(b)
FIG. 2. (Color online) (a) Examples of trapping the incident pulse by the defect with strength $\varepsilon=0.05$, at the following values of the initial peak intensity, $I_{P}: 1.94 \mathrm{GW} / \mathrm{cm}^{2}\left(\mathrm{a}_{1}\right)$, $2.14 \mathrm{GW} / \mathrm{cm}^{2}\left(\mathrm{a}_{2}\right), 2.33 \mathrm{GW} / \mathrm{cm}^{2}\left(\mathrm{a}_{3}\right), 2.59 \mathrm{GW} / \mathrm{cm}^{2}\left(\mathrm{a}_{4}\right)$. (b) The relative width of the trapping interval as a function of the defect's strength, $\varepsilon$.

gratings 22, 36, 37], we now modify the setting, introducing a local defect between the chirped and uniform BG segments, as shown in Fig 1(a). The defect is defined by taking the wavenumber detuning as $\delta^{\prime}(z)=$ $\delta(z)\left\{1+\varepsilon \operatorname{sech}\left[-((z-L / 8) / 0.005)^{2}\right]\right\}$, where $\varepsilon$ is the strength of the defect, and its width is taken as $50 \mu \mathrm{m}$. In comparison with the spatial width of the pulse (the temporal duration of $16 \mathrm{ps}$ corresponds to $4.8 \mathrm{~mm}$ in space) or the total length, $L$, the width of $50 \mu \mathrm{m}$ may indeed be considered as that of a point-like defect. The defect affects the shape of the effective potential, adding a local trapping well to it, as shown in Fig. 1(b).

Noteworthy effects produced by simulations of the setting including the defect are presented in Fig. 2(a), for the defect's strength $\varepsilon=0.05$. The pulse may be stably trapped by the defect (in particular, at peak intensities $I_{P}=2.33 \mathrm{GW} / \mathrm{cm}^{2}$ and $2.59 \mathrm{GW} / \mathrm{cm}^{2}$, at which the $\mathrm{BG}$ soliton would be moving into the uniform $\mathrm{BG}$ in the absence of the defect). The stable trapping is observed in the interval of the peak intensity with relative width $\eta=\left(I_{\max }-I_{\min }\right) /\left(I_{\max }+I_{\min }\right) / 2 \approx 33.2 \%$, where $I_{\max }$ and $I_{\min }$ are the maximum and minimum value of $I_{P}$ for the generation of standing pulse, respectively.

The influence of the defect's strength $\varepsilon$ on the trappinginterval's width $\eta$ was investigated too. As seen in Fig. 2(b), $\eta$ grows linearly with $\varepsilon$. This result is quite natural, as the effective energy of the pulse approaching the defect-induced trapping potential well is proportional to $I_{P}$, and, on the other hand, the depth of the of potential well is proportional to $\varepsilon$. The defect with $\varepsilon<0.01$ cannot trap the pulse, i.e., $\eta(\varepsilon \leq 0.01)=0$. Additional simulations demonstrate that, for the given setting, the decrease of the pulse's temporal width leads to shrinkage and eventually vanishing of the stable-trapping interval, which is a natural consequence of the fact that the spectral width of the pulse gets too broad in comparison with the BG's bandgap.

To observe the Bragg soliton-like pulses passing the attractive defect and moving into the uniform $\mathrm{BG}$, a higher initial peak power is needed. For instance, in Fig. 3(a),

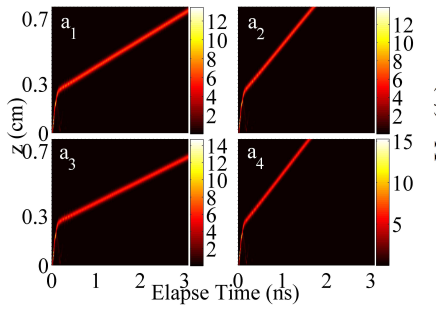

(a)

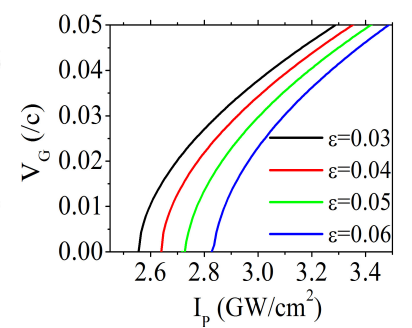

(b)
FIG. 3. (Color online) Free motion of the Bragg soliton in the uniform grating. (a) Trajectories of the motion: $\left(\mathrm{a}_{1}\right)$ for $\varepsilon=0.04, I_{P}=2.65 \mathrm{GW} / \mathrm{cm}^{2} ;\left(\mathrm{a}_{2}\right)$ for $\varepsilon=0.04, I_{P}=2.69$ $\mathrm{GW} / \mathrm{cm}^{2} ;\left(\mathrm{a}_{3}\right)$ for $\varepsilon=0.05, I_{P}=2.74 \mathrm{GW} / \mathrm{cm}^{2} ;\left(\mathrm{a}_{4}\right)$ for $\varepsilon=0.05, I_{P}=2.78 \mathrm{GW} / \mathrm{cm}^{2}$. (b) The soliton's velocity versus the initial peak intensity, $I_{P}$, at different values of the defect's strength.

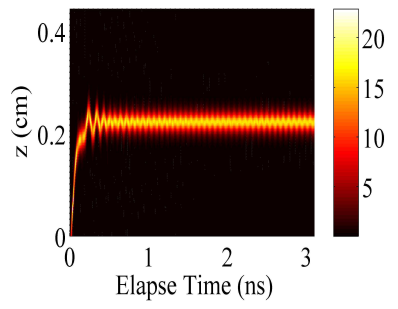

(a)

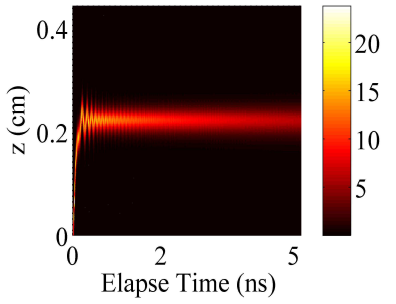

(b)
FIG. 4. (Color online) A typical example of the stable trapping optical pulse with $I_{P}=6.47 \mathrm{GW} / \mathrm{cm}^{2}$, at $\lambda=2 \mu \mathrm{m}$, $C=2.6213 \times 10^{-4} \mathrm{~cm}^{-1}, \Lambda_{0}=292.7 \mathrm{~nm}$ : (a) without the TPA effect; (b) with the TPA effect included $\left(\operatorname{Im}\left[n_{2}\right]=0.2 \times\right.$ $\left.10^{-16} \mathrm{~cm}^{2} / \mathrm{W}\right)$.

with defect's strength $\varepsilon=0.04$, and peak intensities $I_{P}=$ $2.65 \mathrm{GW} / \mathrm{cm}^{2}$ and $2.69 \mathrm{GW} / \mathrm{cm}^{2}$ (panels $\mathrm{a}_{1}$ and $\mathrm{a}_{2}$ ), the pulses keep moving slowly with velocities at $0.005 \mathrm{c}$ and $0.011 c$, respectively. If $\varepsilon$ increases to 0.05 , similar results are obtained for $I_{P}=2.74 \mathrm{GW} / \mathrm{cm}^{2}$ and $2.78 \mathrm{GW} / \mathrm{cm}^{2}$, as seen in panels $\mathrm{a}_{3}$ and $\mathrm{a}_{4}$.

Generally, the slow motion of the Bragg soliton in the uniform BG occurs, with or without the defect, when $I_{P}$ exceeds a certain threshold value, $I_{P}^{(\mathrm{thr})}$. To further elucidate the influence of the defect on the pulse's dynamics, the dependence of velocity $V_{G}$ of the soliton moving inside the uniform BG and $I_{P}$ is depicted in Fig. 3(b), for different values of $\varepsilon$. It is evident that, slightly above the threshold, i.e., at small values of $I_{P}-I_{P}^{\text {(thr) }}$, the velocity grows as $\sqrt{I_{P}-I_{P}^{(\text {thr })}}$, which is typical for depinning phenomena (as the kinetic energy is proportional to the squared velocity).

While, as mentioned above, silicon demonstrates the TPA in the considered spectral region, these losses quickly decrease at $\lambda>1700 \mathrm{~nm}$ [38]. A typical example of stable trapping of the soliton-like pulse with the carrier wavelength $\lambda=2 \mu \mathrm{m}$, predicted by simula- 
tions of Eq. (1) with accordingly modified parameters, is shown in Fig. 4(a). In particular, with the TPA effect included (in this situation, we use a relevant value of its strength, $\left.\operatorname{Im}\left[n_{2}\right]=0.2 \times 10^{-16} \mathrm{~cm}^{2} / \mathrm{W}\right)$, Fig. 4(b) shows that the optical pulse trapped by the defect is maintained well. Further simulations demonstrate that the storage time $\tau$ is nearly inversely proportional to the TPA coefficient, measured in units of $10^{-16} \mathrm{~cm}^{2} / \mathrm{W}$ : $\tau[\mathrm{ns}] \approx 0.8\left(\operatorname{Im}\left[n_{2}\right]\right)^{-1}$. If necessary, $\tau$ can be made indefinitely long by adding a weak local gain compensating the residual loss [39].

In conclusion, we have performed the analysis of the generation of very slow or even standing ultrashort optical pulses in concatenated BGs (Bragg gratings), built as a linearly chirped grating linked to a uniform one, with a defect placed at the junction between them. Physical pa- rameters were taken for silicon, taking care to decrease the detrimental effect of the TPA (two-photon absorption). Systematic simulations have demonstrated that, in agreement with our qualitative analysis, it is possible to provide suitable conditions for the creation of very slow Bragg solitons in this system, or even solitons stably trapped by the defect. The chirped BG segment is necessary for the preparation of an appropriate pulse, built as the right mix of forward and backward wave components, which impinges on the defect and then advances into the uniform grating, unless it gets trapped by the defect. The interval of input peak intensities of the light pulses, which are eventually trapped by the defect, increases linearly with the defect's strength.

This work is supported by Chinese National Basic Research Program (2010CB923204) and Chinese National Natural Science Foundation (11104083, 11204386).
[1] C. J. Chang-Hasnain, P. Ku, J. Kim, and S. Chuang, Proceedings of the IEEE. 91, 1884(2003).

[2] J. T. Mok, C. M. de Sterke, I. C. M. Littler, B. J. Eggleton, Nature Phys. 2, 775(2006).

[3] R. Langenhorst, M. Eiselt, W. Pieper, G. Grobkopf, R. Ludwig, L. Küller, E. Dietrich, H. G. Weber, J. Light. Tech. 14, 324(1996).

[4] A. Kasapi, M. Jain, G. Y. Yin, S. E. Harris, Phys. Rev. Lett. 74, 2447(1995).

[5] C. Liu, Z. Dutton, C. H. Behroozi, L. V. Hau, Nature 409, 490(2001).

[6] J. B. Khurgin, J. Opt. Soc. Am. B. 22, 1062(2005).

[7] Y. A. Vlasov, M. O'Boyle, H. F. Hamann, S. J. McNab, Nature. 438, 65(2005).

[8] H. Altug, J. Vucković, Appl. Phys. Lett. 86, $111102(2005)$.

[9] T. Baba, Nature Phot. 2, 465 (2008).

[10] J. Li, L. O'Faolain, S. A. Schulz, T. F. Krauss, Photonic Nanostructure 10, 589 (2008).

[11] A. E. Kozhekin, G. Kurizki, B. Malomed, Phys. Rev. Lett. 81, 3647 (1998).

[12] J. T. Li and J. Y. Zhou, Opt. Express. 14, 2811 (2006).

[13] W. N. Xiao, J. Y. Zhou, J. P. Prineas, Opt. Express. 11, $3277(2003)$.

[14] R. W. Boyd, J. Opt. Soc. Am. B 28, A38 (2011).

[15] J. B. Khurgin, Adv. Opt. Phot. 2, 287-318 (2010).

[16] B. Corcoran, C. Monat, M. Pelusi, C. Grillet, T. P. White, L. O'Faolain, T. F. Krauss, B. J. Eggleton, and D. J. Moss, Opt. Exp. 18, 7770 (2010).

[17] C. Koos, P. Vorreau, T. Vallaitis, P. Dumon, W. Bogaerts, R. Baets, B. Esembeson, I. Biaggio, T. Michinobu, F. Diederich, W. Freude, and J. Leuthold, Nature Phot. 3, 216 (2009).

[18] J. Atai and B. A. Malomed, Phys. Lett. A. 342, 404 (2005).

[19] D Royston Neill, Javid Atai and Boris A Malomed, J. Opt. A: Pure Appl. Opt. 10, 085105 (2008);
[20] D. N. Christodoulides, R. I. Joseph, Phys. Rev. Lett. 62, 1746(1989).

[21] B. J. Eggleton, R. E. Slusher, C. M. de Sterke, P. A. Krug, J. E. Sipe, , Phys. Rev. Lett. 76, 1627(1996).

[22] P. Y. P. Chen, B. A. Malomed and P. L. Chu, Phys. Rev. E 71, 066601(2005).

[23] A. B. Aceves and S. Wabnitz, Phys. Lett. 141, 37(1989).

[24] W. C. K. Mak, B. A. Malomed, P. L. Chu, J. Mod. Opt. 51, 2141(2004).

[25] W. C. K. Mak, B. A. Malomed, P. L. Chu, Phys. Rev. E. 68, 026609(2003).

[26] K. Qian, L. Zhan, H. Li, X. Hu, J. Peng, L. Zhang, and Y. Xia, Opt. Exp. 17, 22217(2009).

[27] E. N. Tsoy, C. M. de Sterke, Phys. Rev. E. 62, 2882(2000).

[28] D. Janner, G. Galzerano, G. D. Valle, P. Laporta, and S. Longhi, Phys. Rev. E 72, 056605(2005).

[29] C. M. de Sterke, J. E. Sipe, Prog. Opt. 33, 203(1994).

[30] C. M. de Sterke, J. Opt. Soc. Am. B 15, 2660(1998).

[31] R. Shnaiderman, R. S. Tasgal, and Y. B. Band, Opt. Lett. 2438(2011).

[32] L. Poladian, Phys. Rev. E 48, 4758(1993).

[33] W. C. K. Mak, B. A. Malomed, P. L. Chu, J. Opt. Soc. Am. B 20, 725(2003).

[34] M. Dinu, F. Quochi, and H. Garcia, Appl. Phys. Lett. 82, 2954(2003).

[35] O. Boyraz and B. Jalali, Opt. Exp. 12, 5269-5273 (2004).

[36] R. H. Goodman, R. E. Slusher, M. I. Weinstein, J. Opt. Soc. Am. B 19, 1635(2002).

[37] C. M. de Sterke, I. V. Kabakova, I. Uddin, J. Jeyaratnam, and B. A. Malomed, Phys. Rev. A 88, 033825 (2013).

[38] A. D. Bristow, N. Rotenberg, and van H. M. Driel, Appl. Phys. Lett. 90, 191104 (2007).

[39] W. C. K. Mak, B. A. Malomed, and P. L. Chu, Phys. Rev. E 67, 026608 (2003). 